Review Article

\title{
In Vivo Imaging of Neutrophil Extracellular Traps (NETs): Visualization Methods and Outcomes
}

\author{
Sultan Z. Alasmari iD \\ Clinical Laboratory Sciences, Faculty of Applied Medical Sciences, King Khalid University, Abha 62529, Asir Region, \\ Saudi Arabia \\ Correspondence should be addressed to Sultan Z. Alasmari; szaher@kku.edu.sa
}

Received 29 October 2019; Revised 29 December 2019; Accepted 10 January 2020; Published 3 February 2020

Academic Editor: Heide Schatten

Copyright () 2020 Sultan Z. Alasmari. This is an open access article distributed under the Creative Commons Attribution License, which permits unrestricted use, distribution, and reproduction in any medium, provided the original work is properly cited.

\begin{abstract}
Neutrophils comprise the first line of innate immune defense during a host-pathogen interaction. They attack microorganisms directly through three different methods, of which, phagocytosis and degranulation have been known and well-studied for decades. The formation of neutrophil extracellular traps (NETs) is the third and unique method, which was unveiled in 2004. Since then, many studies on NETs have been carried out. However, only few have successfully demonstrated the activity of NETs in vivo. Results of the in vivo studies on NETs have strengthened our understanding of their role in different situations. This review highlights the main in vivo studies, which have contributed in extending our understanding of the role of NETs during infections and diseases, thus indicating their advantages and limitations.
\end{abstract}

\section{Introduction}

Neutrophils are known to play a crucial role in immune defense. They attack microorganisms via three distinct methods, which include phagocytosis, degranulation, and the formation of neutrophil extracellular traps (NETs) [1]. Phagocytosis and degranulation have been known and wellstudied for decades. However, questions still exist about the formation of NETs, which were first described in 2004 by Brinkmann et al. [2]. They demonstrated that stimulated neutrophils undergo unusual morphological changes and produce web-like structures termed NETs, which were composed of DNA, histones, and granular proteins [2]. These structures trap and kill the invading pathogens extracellularly. The formation of NETs is stimulated through the activity of chemicals such as phorbol-12-myristate-13acetate (PAS) and calcium ionophore [2-4]. It has been reported that the formation of NETs is induced by the presence of several pathogens including Gram-positive bacteria such as Staphylococcus aureus [5] and Streptococcus pyogenes [6]; Gram-negative bacteria such as Escherichia coli [7], Shigella flexneri [2], Yersinia enterocolitica [8], and
Yersinia pseudotuberculosis [8]; viruses such as HIV-1 [9]; and other organisms [10].

Furthermore, it has been determined that NETs are involved in a variety of conditions such as cancers and vascular diseases like atherosclerosis, small vessel vasculitis (SVV), and thrombosis [11-14]. The majority of the studies on NET formation have been carried out in vitro. However, in few studies, the formation of NETs in living organisms has been microscopically analyzed. This review focuses on the main in vivo studies, which have been conducted to determine the role of NETs in infections and diseases, thus indicating their advantages and limitations.

\section{The Morphology and Mechanisms of NETs}

NETs are fragile fabrics composed of nuclear components and granules, which trap and, in many cases, kill pathogens extracellularly. High-resolution scanning electron microscopy (SEM) has revealed that this fabric (NETs) is composed of smooth stretches and globular domains aggregating into large threads [2]. The use of immunofluorescence staining methods has revealed that NETs consist of DNA, histones, 


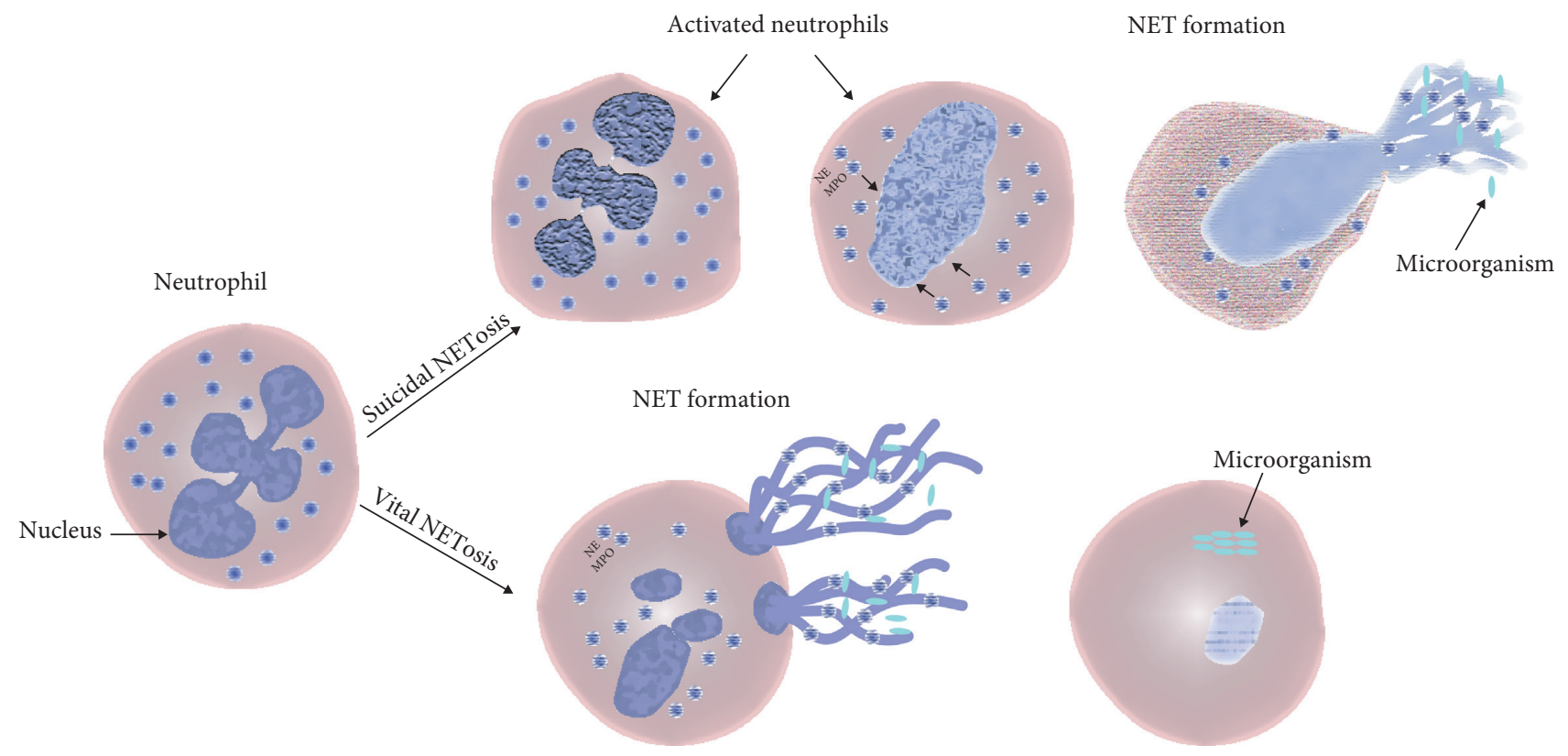

FIGURE 1: Mechanisms of NET formation. Upon activation, phenomenal morphological changes are observed during suicidal NETosis, which is followed by cell rupture and subsequent cell death. Contrarily, in vital NETosis, NETs are released rapidly by the active and functional NET-releasing neutrophils through blebbing of the nuclear envelope and vesicular exportation.

and primary granule proteins such as neutrophil elastase (NE), myeloperoxidase (MPO), and cathepsin G. Lactoferrin and gelatinase are the secondary and tertiary granular portions contained within NETs, respectively [2]. To date, three distinct forms of NET release have been identified.

The first novel mechanism of NET formation involves the occurrence of morphological changes in activated neutrophils. Activated neutrophils tend to flatten and lose the lobules of their nuclei, after which, the chromatin is decondensed, followed lastly by a nuclear detachment of the inner and outer membranes. Besides, the separation of the granules is also observed. After $1 \mathrm{~h}$ of activation, the nuclear envelope breaks into pieces. Finally, the cells round up until the cell membrane ruptures and ejects their internal contents into the extracellular space forming NETs [2]. This type of NET formation is known as a suicidal NET or NETosis (Figure 1). The term NETosis was first coined by Steinberg and Grinstein to describe suicidal NETosis [15]. The second form of NET release is termed as vital NETosis, during which stimulated neutrophils remain active and functional following the NET release (Figure 1) $[16,17]$. The major difference between suicidal and vital NETosis is that the suicidal NET release occurs slowly whereas vital NETosis occurs rapidly $[17,18]$. It has been observed that vital NETosis is induced following bacterial infections while chemical stimuli such as phorbol-12-myristate 13-acetate (PMA) usually induce suicidal NETosis [17]. The mechanisms for NET release are different for suicidal and vital NETosis (Figure 1). Neutrophils stimulated using PMA, uric acid crystals, or Candida albicans induce NET release depending on the induction of NADPH oxidase and activities of elastase and MPO [2, 5, 19-21].

However, bacteria and their products have been found to induce NET release through mechanisms involving toll-like receptors (TLRs) and integrins $[16-18,21,22]$. The third form of NET release is observed upon the activation of neutrophils due to saliva. Neutrophils elicited through the saliva undergo NETosis, which is independent of the activities of NADPH oxidase, elastase, and integrins [21]. Additionally, saliva-induced NETs are resistant to the effects of DNase and have higher bactericidal activities [21]. The second and third types of NETosis are observed in vivo and discussed in detail below.

\section{In Vivo Methods for Visualization and Quantification of NETs}

NET release was first visualized in vitro. The first in vitro study reported that neutrophil lysis results in the formation of fragile web-like structures known as NETs [2]. This discovery has led researchers to carry out extensive studies on the role of NETs in countering infection and during inflammation. The majority of the studies involving NETs have been conducted in vitro. However, a few reports have presented the effect of NET release in vivo. Visualization of NETs in vitro is dependent on the composition of their histones, MPO, neutrophil elastase, and DNA. Despite visualizing NETs successfully in vitro, questions on their effects in vivo have remained unclear due to the limited number of in vivo studies. Most of the articles published to date on in vivo studies involving NET quantification have focused on their direct visualization using advanced microscopic techniques such as spinning-disk confocal intravital [16, 23-25] or two-photon microscopy [26, 27].

Fluorescent labeling of microorganisms is another method used for the direct visualization of pathogen interaction with NET-releasing neutrophils and NET in vivo $[16,23]$. NETs have been directly visualized in vivo using 
several methods such as staining of the extracellular DNA using membrane-impermeable DNA dyes [16, 23-28] and fluorescently labeled antibodies specific to the NET components such as histone and neutrophils elastase $[16,23-25,27]$. Additionally, cell-permeable and cell-impermeable DNA dyes have been used in combination in some studies to distinguish between the intercellular and extracellular DNA of the cells [16]. Cell-permeable DNA dyes have been administrated to enable nuclear imaging [16]. To investigate the interaction with the presence or absence of NETs in vivo, another method involving the use of DNase has been employed, which has verified that NETs are composed of DNA and also enabled evaluation of its degradation $[23-25,27]$. Some studies have used an inhibitor of neutrophil elastase to demonstrate the degradation of NET $[24,25]$. Neutrophil depletion in vivo has been employed to determine the importance of neutrophils as a source of NET through treatment using neutrophil-specific antibodies [23-25]. NETs have been quantified by determining their sizes using a velocity imaging software [16] and the ImageJ software $[23,24]$. NETs were quantified in vivo by counting their numbers in 10 randomly selected fields of intravascular spaces, hepatic sinusoids, and pulmonary capillaries using intravital multiphoton microscopy (MPM) imaging. The total number of NETs in these 10 fields was summed and expressed as the number of NETs present per field of view [27]. The behaviors of neutrophils were examined by tracking an individual neutrophil, and its velocity, distance, and displacement were determined using the Volocity software [16]. Interestingly, all in vivo studies mentioned above have been carried out using mouse models $[16,23-28]$.

\section{In Vivo Studies on NET Formation}

Extensive in vitro studies conducted on the formation of NETs and their relationship with diseases have raised many questions about their role in vivo. In vivo research on NETs is rendered complicated due to their fragility. However, some studies involve the use of complex methods to visualize NETs and determine their roles in vivo. Here, data from some promising in vivo studies, which have been published previously, has been summarized. Live cell imaging is the method of choice to directly visualize the morphologies and behaviors of NETs. McDonald et al. monitored the recruitment of neutrophils to the liver sinusoids during bacterial sepsis and visualized NET formation using live cell imaging and dual laser multichannel spinning-disk microscopy, respectively [23]. To visualize NETs in vivo, a cellimpermeable DNA dye was used to stain the extracellular web-like DNA structure created by the neutrophils within the lumens of the sinusoids.

Dissolution of the dye following the administration of DNase confirmed that the observed structures were composed of DNA. Additionally, fluorescently labeled antibodies specific to the constituents of NETs, which included histones and neutrophil elastase, were intravenously infused to confirm that the web-like DNA structures were NETs. McDonald et al. have demonstrated that NETs could form a large extracellular web within the liver sinusoids even under flow conditions. However, it was observed that they were not attached stably to the walls of the vessels of venules with the larger and higher shear compared to those of the slowflowing liver sinusoids. A research by $\mathrm{McDonald}$ et al. revealed that the interaction between NETs and bacteria could be monitored in vivo upon infusion of fluorescently labeled E. coli into mice. Visualizing the formation of NETs in vivo has enabled us to confirm that they eliminate bacteria from dissemination [23].

Yipp et al. have developed assays to demonstrate the role of NETs in vivo using cell-impermeable/permeable DNA and fluorescently conjugated antibodies [16]. In the first assay, the formation of the extracellular web-like DNA structure was visualized in vivo using a cell-impermeable DNA dye infused $30 \mathrm{~min}$ prior to the administration of $S$. aureus. The second assay was designed using antibodies specific to the components of NETs such as histones and neutrophil elastase. Rapid release of histones and neutrophil elastase was found to occur in response to infection by $S$. aureus and S. pyogenes [16]. This result was contrary to that observed in the early in vitro study involving NETs, which revealed that neutrophils form NETs within hours of subjecting them to the inciting stimuli [5]. The third assay was designed using cell-permeable DNA and allowed direct visualization of the nucleus of intact neutrophils and quantification of the ratio of extracellular to intracellular DNA during sterile inflammation and infection [16]. Additionally, the results of the third assay helped determine the fate of the NET-releasing neutrophils. It was observed that NET-releasing neutrophils die following the NET release in vitro [5]. However, in vivo imaging of NETosis revealed that the NET-releasing neutrophils remain active and functional following the NET release [16].

Formation of NETs has also been monitored in vivo during sepsis. Widespread deposition of NETs was observed in murine livers upon subjecting them to sepsis induced by cecal ligation and puncture (CLP) [24]. NETs were observed in vivo through the intravascular administration of a cellimpermeable DNA dye and using fluorescently labeled antibodies against granular proteins [24]. It has been demonstrated that the intravascular injection of fluorescently labeled antibodies against the histone could stably and effectively stain NETs in vivo $[23,24]$. A reduction in the amount of extracellular DNA was observed in mice subjected to CLP, which was stained using a cell-impermeable DNA dye in the liver and lung following the administration of an antibody for neutrophil depletion, DNase, and a neutrophil elastase inhibitor [24]. Interestingly, using a murine model, Cools-Lartigue et al. investigated the mechanism by which NETs interacted with tumor cells in vivo following their intravascular injection. They determined that tumor cells were trapped within the NETs present in the hepatic sinusoids of living mice and pulmonary capillaries ex vivo [24].

NETs have also been characterized in vivo in various organs of a murine sepsis model [27]. Tanaka et al. administrated a cell-impermeable DNA dye and anti-histone or anti-neutrophil elastase antibodies intravenously to GFP 
mice following lipopolysaccharide (LPS) stimulation to visualize the behavior of NETs in various organs. Firstly, different structures of NETs in the postcapillary venules of the cecum were observed, which were as follows: (1) reticular structures anchored to the leukocytes, (2) reticulolinear structures, (3) spotlike structures anchored to the leukocytes, (4) membranous structures on the surface of leukocytes, and (5) linear structures anchored to the leukocytes [27]. Surprisingly, the same study revealed that the occurrence of NETs in vivo is a rare event compared to the leukocyte-endothelial interaction [27]. Additionally, the presence of leukocytes was observed in the postcapillary venules of the cecum in mice treated with LPS, in which cytoplasmic vacuoles adhering to the vascular endothelium were found at the subcellular level. However, the occurrence of these characteristic leukocytes was less frequent in the arterioles and hepatic sinusoids [27]. Interestingly, few of these leukocytes were found to consist of cytoplasmic vacuoles, which released the NETs. It was unclear whether these cells were active or dead. However, the authors have stated that these cells might have undergone a suicidal form of NETosis. Secondly, the authors have reported a feature of NETs in the hepatic sinusoids of the liver, in which spotlike structures anchored to the leukocytes, cell-free DNA fragments, and those within platelet aggregates were observed in mice treated with LPS [27].

Tanaka et al. have reported the presence of few NETs in the hepatic sinusoids, an observation contrary to the previous findings of studies conducted in vivo on NETs by McDonald et al. [23, 24, 27]. Circulating cell-free NETs, which are characterized as fragmented or cotton-like structures, were detected in the blood of either hepatic sinusoids of the liver or postcapillary venules of the cecum following intraperitoneal administration of LPS [27]. It has been elucidated that the rate of blood flow might have played a role in determining the shape of cell-free NETs. Additionally, Tanaka et al. have reported that NETs could interact with the platelets, endothelial cells, or leukocyte-platelet aggregates in vivo [27]. This finding supports those revealed in previous reports on the interaction of NETs with platelets [29] and endothelial cells [30, 31]. However, further investigations are needed to determine the direct impact of NETs on these phenomena in vivo. However, this finding could be used to support those determined in the previous studies involving the interaction of NETs with platelets and endothelial cells [29].

Moreover, Kolaczkowska and the group determined that NETs could persist for up to $24 \mathrm{~h}$ in the liver vasculature after infection [25]. They revealed that the NETs are robust at $4 \mathrm{~h}$ and $9 \mathrm{~h}$ following infection with methicillin-resistant $S$. aureus (MRSA). Besides, the administration of DNase removes the extracellular DNA precisely but not the other NET components lining the walls of the liver sinusoids [25]. However, the presence of other components of NETs, such as histones and neutrophil elastase, might cause tissue injury even in the presence of a DNase inhibitor. Kolaczkowska et al. showed that NETs, not the pathogens, are entirely responsible for the liver damage inflicted after the infection with MRSA [25]. It has been determined that von Willebrand factor (VWF), which is normally associated with hemostasis and has been found to bind histones [32], could interact with NETs in vivo in the liver vasculature [25]. Adherence of histones and elastase to the sinusoidal endothelium and binding of the DNA to the vessel wall in mice pretreated with VWF-blocking antibodies could be prevented and reduced, respectively, under these conditions [25].

\section{Conclusions}

In vivo studies on NETosis have been conducted using advanced technology. These technologies have helped scientists understand the behavior of NETs at several sites in the animal models. The majority of the in vivo studies, if not all, have been conducted using mouse models, in which the behavior of NETs following the encounter with pathogens or tumor cells has been monitored. Although the use of mouse models has tremendously improved our understanding of the role of NETs in vivo, such studies have certain limitations. The in vivo studies of NETosis discussed in this review have involved the use of extensive surgical approaches in mice, followed by the administration of fluorescently labeled microorganisms and conjugated antibodies to generate infection and mark the NETs or neutrophils, respectively. These procedures might have impacted generated data, since they are invasive and do not take into account the fact that the animal is complete and intact. The use of other animal models such as zebrafish could help reduce the limitations of these studies. The zebrafish model is a powerful system used for the in vivo imaging of neutrophils because it complements the research conducted on mice and humans and also combines genetic modulation and in vivo imaging $[33,34]$. Zebrafish has recently been accepted as a model system for the imaging of immune cells during wound healing and infection [35-38]. Imaging cell behaviors using a zebrafish model enables investigators to noninvasively examine the function of immune cells for an extended duration by regarding the whole animal as the study system [39]. The main advantage of using this model is that it enables researchers to fluorescently mark the transgenic lines within specific parts of the cells, which can consequently be visualized clearly during the early development of the zebrafish due to the optical transparency of its embryo [33, 39].

\section{Conflicts of Interest}

The author declares no conflicts of interest.

\section{Acknowledgments}

The author is grateful to Volker Brinkmann, Fahad Alshehri, and Philip Raines for critically reading this paper. The author would also like to thank Editage (http://www.editage.com) for English language editing.

\section{References}

[1] C. Rosales, "Neutrophil: a cell with many roles in inflammation or several cell types?" Frontiers in Physiology, vol. 9, p. 113, 2018. 
[2] V. Brinkmann, U. Reichard, C. Goosmann et al., "Neutrophil extracellular traps kill bacteria," Science, vol. 303, no. 5663, pp. 1532-1535, 2004.

[3] L. Barrientos, V. Marin-Esteban, L. d. Chaisemartin et al., "An improved strategy to recover large fragments of functional human neutrophil extracellular traps," Frontiers in Immunology, vol. 4, p. 166, 2013.

[4] M. J. Kaplan and M. Radic, "Neutrophil extracellular traps: double-edged swords of innate immunity," The Journal of Immunology, vol. 189, no. 6, pp. 2689-2695, 2012.

[5] T. A. Fuchs, U. Abed, C. Goosmann et al., "Novel cell death program leads to neutrophil extracellular traps," The Journal of Cell Biology, vol. 176, no. 2, pp. 231-241, 2007.

[6] J. T. Buchanan, A. J. Simpson, R. K. Aziz et al., "DNase expression allows the pathogen group A Streptococcus to escape killing in neutrophil extracellular traps," Current Biology, vol. 16, no. 4, pp. 396-400, 2006.

[7] N. Grinberg, S. Elazar, I. Rosenshine, and N. Y. Shpigel, "-Hydroxybutyrate abrogates formation of bovine neutrophil extracellular traps and bactericidal activity against mammary pathogenic Escherichia coli," Infection and Immunity, vol. 76, no. 6, pp. 2802-2807, 2008.

[8] S. Casutt-Meyer, F. Renzi, M. Schmaler, N. J. Jann, M. Amstutz, and G. R. Cornelis, "Oligomeric coiled-coil adhesin YadA is a double-edged sword," PLoS One, vol. 5, no. 12, Article ID e15159, 2010.

[9] T. Saitoh, J. Komano, Y. Saitoh et al., "Neutrophil extracellular traps mediate a host defense response to human immunodeficiency virus-1," Cell Host \& Microbe, vol. 12, no. 1, pp. 109-116, 2012.

[10] V. Brinkmann and A. Zychlinsky, "Neutrophil extracellular traps: is immunity the second function of chromatin?," The Journal of Cell Biology, vol. 198, no. 5, pp. 773-783, 2012.

[11] K. H. Lee, A. Kronbichler, D. D.-Y. Park et al., "Neutrophil extracellular traps (NETs) in autoimmune diseases: a comprehensive review," Autoimmunity Reviews, vol. 16, no. 11, pp. 1160-1173, 2017.

[12] J. Park, R. W. Wysocki, Z. Amoozgar et al., "Cancer cells induce metastasis-supporting neutrophil extracellular DNA traps," Science Translational Medicine, vol. 8, no. 361, Article ID 361ra138, 2016.

[13] R. Oklu, R. A. Sheth, K. H. K. Wong, A. H. Jahromi, and H. Albadawi, "Neutrophil extracellular traps are increased in cancer patients but does not associate with venous thrombosis," Cardiovascular Diagnosis and Therapy, vol. 7, no. S3, pp. S140-S149, 2017.

[14] D. Dąbrowska, E. Jabłońska, M. Garley, J. SawickaPowierza, and K. Nowak, "The phenomenon of neutrophil extracellular traps in vascular diseases," Archivum Immunologiae et Therapiae Experimentalis, vol. 66, no. 4, pp. 273-281, 2018.

[15] B. E. Steinberg and S. Grinstein, "Unconventional roles of the NADPH oxidase: signaling, ion homeostasis, and cell death," Science's STKE, vol. 2007, no. 379, p. pe11, 2007.

[16] B. G. Yipp, B. Petri, D. Salina et al., "Infection-induced NETosis is a dynamic process involving neutrophil multitasking in vivo," Nature Medicine, vol. 18, no. 9, pp. 1386-1393, 2012.

[17] B. G. Yipp and P. Kubes, "NETosis: how vital is it?," Blood, vol. 122, no. 16, pp. 2784-2794, 2013.

[18] F. H. Pilsczek, D. Salina, K. K. H. Poon et al., "A novel mechanism of rapid nuclear neutrophil extracellular trap formation in response to Staphylococcus aureus," The Journal of Immunology, vol. 185, no. 12, pp. 7413-7425, 2010.
[19] V. Papayannopoulos, K. D. Metzler, A. Hakkim, and A. Zychlinsky, "Neutrophil elastase and myeloperoxidase regulate the formation of neutrophil extracellular traps," The Journal of Cell Biology, vol. 191, no. 3, pp. 677-691, 2010.

[20] C. Schauer, C. Janko, L. E. Munoz et al., "Aggregated neutrophil extracellular traps limit inflammation by degrading cytokines and chemokines," Nature Medicine, vol. 20, no. 5, pp. 511-517, 2014.

[21] T. Mohanty, J. Sjögren, F. Kahn et al., "A novel mechanism for NETosis provides antimicrobial defense at the oral mucosa," Blood, vol. 126, no. 18, pp. 2128-2137, 2015.

[22] S. R. Clark, A. C. Ma, S. A. Tavener et al., "Platelet TLR4 activates neutrophil extracellular traps to ensnare bacteria in septic blood," Nature Medicine, vol. 13, no. 4, pp. 463-469, 2007.

[23] B. McDonald, R. Urrutia, B. G. Yipp, C. N. Jenne, and P. Kubes, "Intravascular neutrophil extracellular traps capture bacteria from the bloodstream during sepsis," Cell Host \& Microbe, vol. 12, no. 3, pp. 324-333, 2012.

[24] J. Cools-Lartigue, J. Spicer, B. McDonald et al., "Neutrophil extracellular traps sequester circulating tumor cells and promote metastasis," Journal of Clinical Investigation, vol. 123, no. 8, pp. 3446-3458, 2013.

[25] E. Kolaczkowska, C. N. Jenne, B. G. J. Surewaard et al., "Molecular mechanisms of NET formation and degradation revealed by intravital imaging in the liver vasculature," Nature Communications, vol. 6, no. 1, p. 6673, 2015.

[26] Y. Döring, H. D. Manthey, M. Drechsler et al., "Auto-antigenic protein-DNA complexes stimulate plasmacytoid dendritic cells to promote atherosclerosis," Circulation, vol. 125, no. 13, pp. 1673-1683, 2012.

[27] K. Tanaka, Y. Koike, T. Shimura et al., "In vivo characterization of neutrophil extracellular traps in various organs of a murine sepsis model," PLoS One, vol. 9, no. 11, Article ID e111888, 2014.

[28] M.-L. von Brühl, K. Stark, A. Steinhart et al., "Monocytes, neutrophils, and platelets cooperate to initiate and propagate venous thrombosis in mice in vivo," The Journal of Experimental Medicine, vol. 209, no. 4, pp. 819-835, 2012.

[29] T. A. Fuchs, A. Brill, D. Duerschmied et al., "Extracellular DNA traps promote thrombosis," Proceedings of the $\mathrm{Na}$ tional Academy of Sciences, vol. 107, no. 36, pp. 15880-15885, 2010.

[30] M. Saffarzadeh, C. Juenemann, M. A. Queisser et al., "Neutrophil extracellular traps directly induce epithelial and endothelial cell death: a predominant role of histones," PLoS One, vol. 7, no. 2, Article ID e32366, 2012.

[31] C. Carmona-Rivera, W. Zhao, S. Yalavarthi, and M. J. Kaplan, "Neutrophil extracellular traps induce endothelial dysfunction in systemic lupus erythematosus through the activation of matrix metalloproteinase-2," Annals of the Rheumatic Diseases, vol. 74, no. 7, pp. 1417-1424, 2015.

[32] C. M. Ward, T. J. Tetaz, R. K. Andrews, and M. C. Berndt, "Binding of the von Willebrand factor A1 domain to histone," Thrombosis Research, vol. 86, no. 6, pp. 469-477, 1997.

[33] E. A. Harvie and A. Huttenlocher, "Neutrophils in host defense: new insights from zebrafish," Journal of Leukocyte Biology, vol. 98, no. 4, pp. 523-537, 2015.

[34] K. M. Henry, C. A. Loynes, M. K. B. Whyte, and S. A. Renshaw, "Zebrafish as a model for the study of neutrophil biology," Journal of Leukocyte Biology, vol. 94, no. 4, pp. 633-642, 2013.

[35] S. A. Renshaw, C. A. Loynes, D. M. I. Trushell, S. Elworthy, P. W. Ingham, and M. K. B. Whyte, "A transgenic zebrafish 
model of neutrophilic inflammation," Blood, vol. 108, no. 13, pp. 3976-3978, 2006.

[36] J. R. Mathias, B. J. Perrin, T.-X. Liu, J. Kanki, A. T. Look, and A. Huttenlocher, "Resolution of inflammation by retrograde chemotaxis of neutrophils in transgenic zebrafish," Journal of Leukocyte Biology, vol. 80, no. 6, pp. 1281-1288, 2006.

[37] F. Ellett, L. Pase, J. W. Hayman, A. Andrianopoulos, and G. J. Lieschke, "mpeg1 promoter transgenes direct macrophage-lineage expression in zebrafish," Blood, vol. 117, no. 4, pp. e49-e56, 2011.

[38] F. Ellett, V. Pazhakh, L. Pase et al., "Macrophages protect Talaromyces marneffei conidia from myeloperoxidase-dependent neutrophil fungicidal activity during infection establishment in vivo," PLoS Pathogens, vol. 14, no. 6, Article ID e1007063, 2018.

[39] C. Hall, M. V. Flores, K. Crosier, and P. Crosier, "Live cell imaging of zebrafish leukocytes," in Methods in Molecular Biology, vol. 546, pp. 255-271, Springer, Berlin, Germany, 2009. 